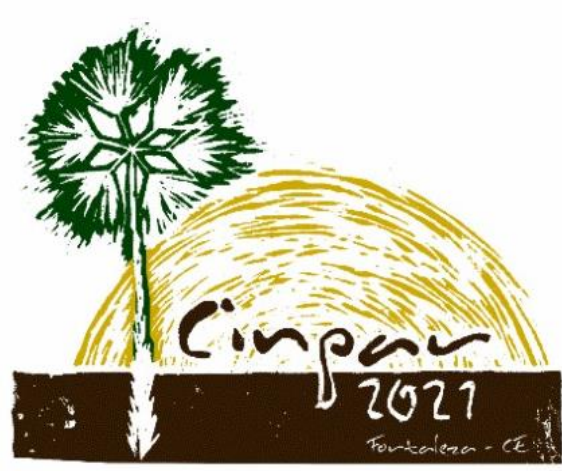

XVII Congresso Internacional sobre Patologia e

Reabilitação das Construções

XVII Congreso Internacional sobre Patología y Rehabilitación de las Construcciones

XVII International Conference on Pathology and Constructions Rehabilitation

FORTALEZA (Brasil), 3 a 5 de junho de 2021

https://doi.org/10.4322/CINPAR.2021.076

\title{
Influência das ações de manutenção na durabilidade de revestimentos de fachadas
}

\section{Influence of maintenance actions on the durability of facade cladding}

\author{
Alan Victor DIAS ${ }^{1}$, Jéssica Siqueira de SOUZA ${ }^{2}$, Yuri Sotero Bomfim FRAGA ${ }^{3}$,Larissa Carvalho de SOUSA \\ Matheus L. M. NASCIMENTO ${ }^{5}$ \\ ${ }^{1}$ Universidade de Brasília, Brasília, Brasil, alanvictor.dias@gmail.com \\ ${ }^{2}$ Universidade de Brasília, Brasília, Brasil, jss.siqueira@gmail.com \\ ${ }^{3}$ Universidade de Brasília, Brasília, Brasil, yurisotero.engcivil@gmail.com \\ ${ }^{4}$ Universidade de Brasília, Brasília, Brasil, larissacarsousa@gmail.com \\ ${ }^{5}$ Instituto de Educação Superior de Brasília, Brasília, Brasil, leoni.matheus@gmail.com
}

\begin{abstract}
Resumo: As edificações e seus sistemas tendem a deteriorar-se ao longo do tempo por isso, as ações de manutenção são imprescindíveis no âmbito da durabilidade com vistas no alcance da vida útil de projeto de uma edificação. Dentro desse escopo, o presente artigo pretende analisar a frequência de danos e como as ações de manutenção podem prolongar a vida útil funcional do sistema de revestimento de fachadas. Por meio de dados bibliográficos e experimentais de inspeção de fachadas, neste estudo é identificado quais os danos mais frequentes e listado quais as possíveis ações de manutenção em sistemas de revestimento cerâmico em fachadas que permitam melhorar o desempenho e durabilidade do sistema. Os resultados evidenciam a importância das ações de manutenção em fachadas, principalmente se tratando do descolamento cerâmico e indicam a periodicidade de ações que devem ser realizadas nas fachadas durante a vida útil.
\end{abstract}

Palavras-chave: Durabilidade; Vida útil; Manutenção; Revestimento de Fachadas.

\begin{abstract}
Buildings and their systems tend to deteriorate over time. Therefore, maintenance actions are essential in the scope of durability with a view to reaching the design life of a building. Within this scope, this article intends to analyze the frequency of defects and how maintenance actions can extend the functional life of the facade cladding system. Through bibliographic and experimental data on facade inspection, this study identifies the most frequent defect and lists the possible maintenance actions for ceramic cladding systems on facades that allow improving the system's performance and durability. The results show the importance of maintenance actions on facades, mainly when it comes to the ceramic cladding detachment and indicates the frequency of actions that must be carried out on facades during their service life.
\end{abstract}

Keywords: Durability; Service Life; Maintenance; Facade Cladding. 


\section{Introdução}

Após a construção, a tendência natural de qualquer edificação é perder o desempenho ao longo do tempo, devido ao processo denominado degradação. Desde o início do uso do edifício, fatores como a qualidade dos componentes, nível de projeto, nível de execução, ambiente interno e externo e condições de uso atuam negativamente sobre a vida útil da edificação (ISO 15686-7, 2006). Entretanto, alguns autores afiram que as ações de manutenção e a frequência destas é um fator que afeta positivamente sobre a vida útil, tais como Duling et al. (2008) e Souza et al. (2018). O desempenho dos sistemas da edificação consiste no comportamento em uso e na capacidade de atendimento das necessidades dos usuários (NBR 15575-1, 2013; NBR 5674, 2012).

Dentro do contexto de durabilidade das edificações, a durabilidade consiste na capacidade do sistema desempenhar suas funções ao longo do tempo sob condições de uso e manutenção até um estado limite de utilização (NBR 15575-1, 2013). A norma brasileira NBR 5674 (2012) define a manutenção como o conjunto de atividades a serem realizadas para conservar ou recuperar a capacidade funcional da edificação e seus respectivos sistemas constituintes. A Figura 1 ilustra o comportamento do desempenho ao longo do tempo quando há ações periódicas de manutenção. As ações de manutenção elevam o desempenho do sistema e prolonga a vida últil da edificação, entretanto o aumento da vida útil depende do tipo de intervenção adotada.

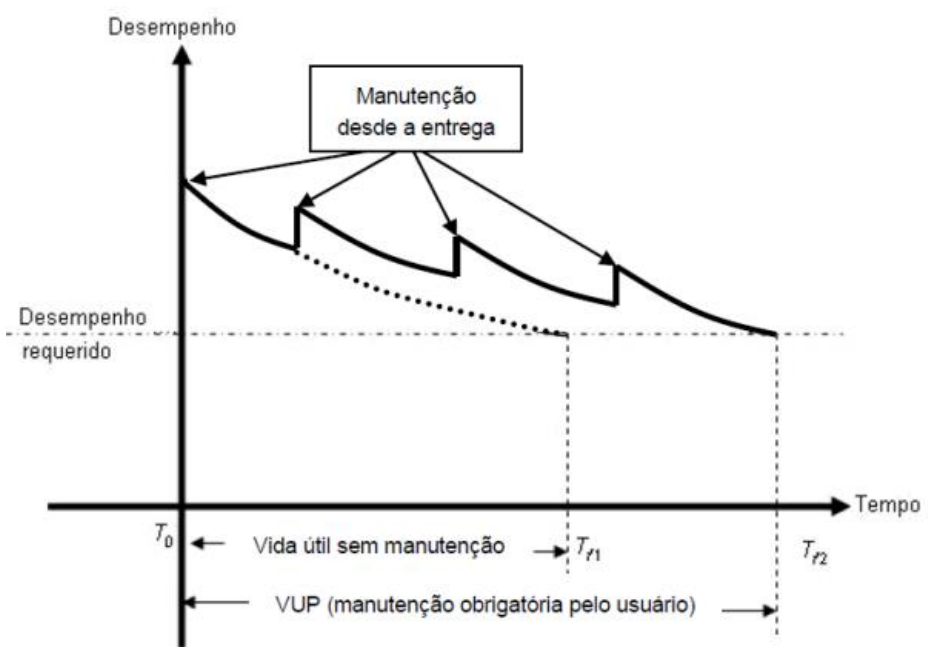

Figura 1 - Desempenho de um elemento ou sistema ao longo do tempo (ABNT NBR 15575-1, 2013)

Os sistemas de revestimento cerâmicos de fachadas são um dos mais afetados por agentes de degradação, podendo variar de intesidade conforme a cidade e de acordo com as características do microclima. A fachada de uma edificação apresenta funções estéticas, que promovem valorização do imóvel, e funções técnicas como proteção e impermeabilização. Quando as funções da fachada não são cumpridas, o desempenho desta pode estar comprometido. Sendo assim para que a edificação não tenha perdas funcionais e desvalorização do empreendimento, é necessário realizar periodicamente ações de manutenção a fim de garantir o desempenho do sistema.

Dentro deste contexto, a presente pesquisa busca contribuir no entendimento da influência das ações de manutenção na durabilidade de revestimentos de fachadas, por meio da aplicação do método de mensuração de degradação. Além disso, utiliza o indicador de degradação Fator de Danos (FD) para mensurar o nível de condição da anomalia, o que possibilita a listagem de possíveis ações de manutenção e como estas podem influenciar na durabilidade do sistema como um todo. As ações de manutenção listadas prevêem horizontes de ações anuais, bienais e a cada três anos de forma que as mesmas garantam o cumprimento ou mesmo permitam o prolongamento da vida útil do sistema. 


\section{Metodologia}

Para esta pesquisa foi utilizado um base de dados de edifícios situados no Distrito Federal, unidade federativa do Brasil, onde está localizada Brasília, a capital federal. A amostra de estudos contempla por volta de 80.000 metros de fachada, as quais foram submetidos ao método de Mensuração de Degradação e depois avaliado o indicador de degradação Fator de Danos (Piazzarollo et al., 2019).

\subsection{Método de Mensuração de Degradação}

A mensuração da degradação de forma quantitativa pode ser obtida pela aplicação do Método de Mensuração de Degradação (MMD). Este método foi desenvolvido por pesquisadores da Universidade de Brasília, Brasil, com o objetivo de avaliar a degradação de fachadas (Pinheiro et al., 2017). As etapas para a mensuração e avaliação da degradaçao vão desde a inspeção do edifício até a obtenção dos indicadores de degradação. A Figura 2 ilustra as etapas para a quantificação da degradação.

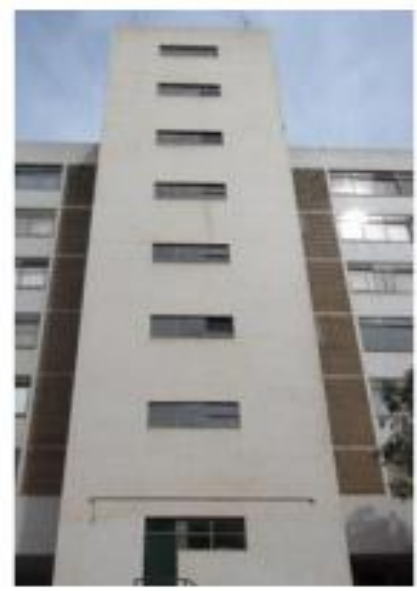

(A) Foto geral da fachada

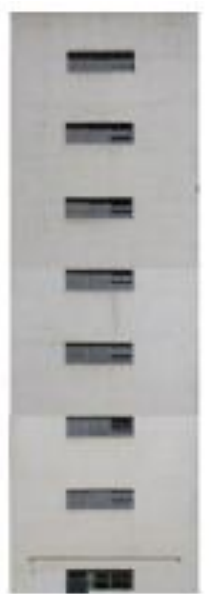

(B) Foto ortogonalizada

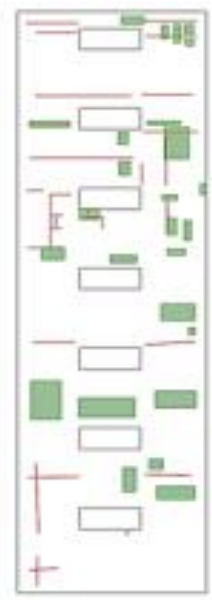

(C) Croqui com mapeamento de danos

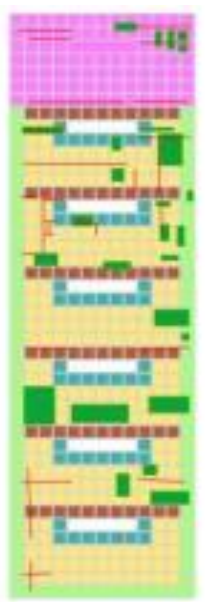

(D) Sobreposição da malha e definição das zonas.

Figura 2 - Procedimentos para aplicação do MMD(Souza, 2019)

A quantificação da degradação é obtida em extensão de área após a sobreposição de uma malha ao mapeamento de danos com dimensões equivalentes à $50 \mathrm{~cm} \times 50 \mathrm{~cm}$. Em sistemas de revestimento cerâmico as anomalias contabilizadas são descolamento cerâmico, fissuração, falha no rejunte e eflorescência, pois estas são as anomalias mais comuns neste tipo de revestimento (Silva et al. 2014).

\subsection{Indicador de degradação}

Após a quantificação da degradação é possivel obter alguns indicadores de degradação. Segundo Souza (2016), apesar de não levar em conta a influência individual de cada tipo de anomalia, o Fator de Danos (FD) avalia a extensão dos danos em função da área total da fachada inspecionada. Este indicador permite avaliar o comportamento da degradação ao longo do tempo e pode ser obtido pela equação 1.

$$
F D=\frac{\sum A_{d(n)}}{A_{t}}
$$

Em que FD é o indicador de degradação Fator de Danos, $A_{d(n)}$ é a área de determinada anomalia (n) em $m^{2} e$ $A_{t}$ é a área total da amostra de fachada em $\mathrm{m}^{2}$. Segundo Souza (2016) o FD seria um parâmetro inicial do estudo do fenômeno de degradação. Ainda existem outros índices de degradação que podem ser utilizados nos estudos desses fenômenos, como o FDG, mas para este trabalho apenas o FD é suficiente.

Os níveis de condição permitem agrupar e classificar o estado de degradação da fachada analisada. A análise do estado de degradação permite verificar a condição da anomalia e assim realizar associação com as ações de manutenção a serem realizadas, além de indicarem a se há urgência para o reparo. 0 quadro 1 descreve os níveis de condição e os critérios para a classificação do indicador de degradação FD. 
Quadro 1 - Descrição e critérios dos níveis de condição (Adaptado de Souza, 2019)

\begin{tabular}{|c|c|l|c|c|}
\hline Condição & \multicolumn{3}{|c|}{ Descrição } & FD \\
\hline A & $\begin{array}{c}\text { Condição boa } \\
\text { (aceitável) }\end{array}$ & $\begin{array}{l}\text { Condição caracterizada por apresentar boas } \\
\text { condições. A incidência de anomalias não prejudica a } \\
\text { funcionalidade e durabilidade do sistema. }\end{array}$ & $0.00-0.19$ \\
\hline B & $\begin{array}{c}\text { Condição de } \\
\text { degradação } \\
\text { pontual }\end{array}$ & $\begin{array}{l}\text { Condição caracterizada pela presença de anomalias } \\
\text { pontuais que prejudicam a funcionalidade. } \\
\text { Entretanto não prejudicam a durabilidade e } \\
\text { segurança do sistema. }\end{array}$ & $0.20-0.84$ \\
\hline C & $\begin{array}{c}\text { Condição de } \\
\text { estado limite de } \\
\text { serviço }\end{array}$ & $\begin{array}{l}\text { Condição caracterizada pela presença generalizada } \\
\text { de anomalias que prejudicam a funcionalidade e a } \\
\text { durabilidade do sistema. }\end{array}$ & $0.85-1.39$ \\
\hline D & $\begin{array}{c}\text { Condição de } \\
\text { estado limite } \\
\text { último }\end{array}$ & $\begin{array}{l}\text { Condição caracterizada pela presença generalizada } \\
\text { de anomalias que prejudicam a funcionalidade e a } \\
\text { durabilidade do sistema, além de reduzir } \\
\text { significativamente os níveis de segurança. }\end{array}$ & $>1.40$ \\
\hline
\end{tabular}

\section{Análise de resultados}

A aplicação do MMD à edificios residenciais e obtenção do FD permitiram realizar análises relacionadas aos níveis de condição propostos por Souza (2019). O descolamento cerâmico é a anomalia mais comum encontrado nos sistemas de revestimentos cerâmicos de fachadas. A Figura 3 mostra os valores dos níveis de degradação referente ao descolamento cerâmico de acordo com a idade de cada revestimento.

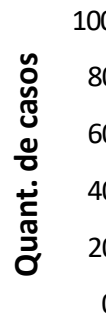

(a)
00
80
60
40
20
0

\begin{abstract}
Condição $A$
\end{abstract}
$(0,0-0,19)$

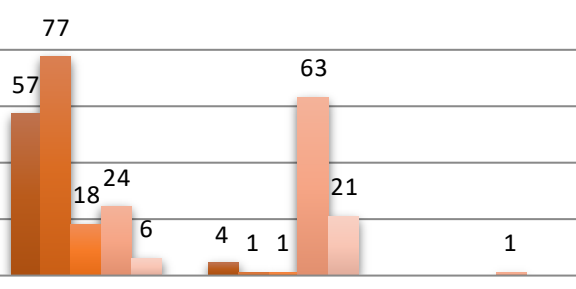

Condição B Condição $C$ $(0,20-0,84) \quad(0,85-1,39)$
$0-10$

- 10-20

- 20-30

$\square 30-40$

$40-50$
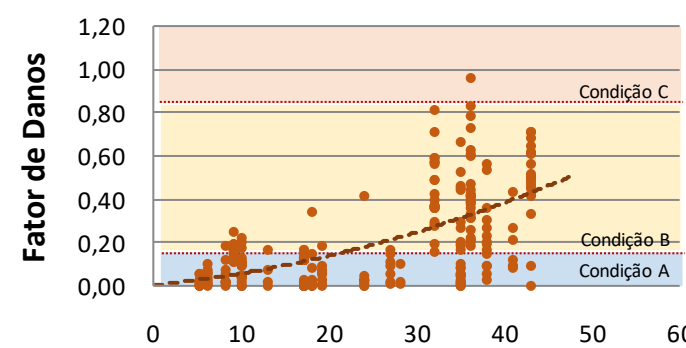

(b)

Idade (anos)

Figura 3 - Níveis de condição referente ao descolamento cerâmico: (a) Ocorrência de casos por faixa de idade e (b) Fator de Danos ao longo do tempo.

A maior quantidade de casos se apresentam na Condição $A$, isto indica que a maioria dos casos apresentam valores de FD de descolamento cerâmico inferior a 0,19. Entretanto, nota-se que há uma redução considerável na quantidades de casos na Condição $A$ das idades inferiores a 20 anos para as idades mais avançadas. $O$ contrário pode ser observado na Condição $B$, em que há poucos casos nas idades mais jovens e maior quantidade nas idades superiores a 30 anos. Segundo Souza (2019), na Condição B são sugeridas a realização de manutenção preditiva, isto é, manutenções periódicas para avaliarem o sistema.

Embora haja uma elevada quantidade de casos na Condição A, observa-se que o FD aumenta para as idades mais avançadas (Figura 3 b). Nota-se que até os 30 anos de idade a maioria dos casos pertencem a Condição A e que após essa idade a Condição B passa a ser a dominante. Isto indica que pequenos danos, quando não tratados, podem tornar-se mais graves com o tempo. A elevada incidência de descolamento cerâmico nas idades iniciais podem ser justificada pela falha nas etapas de projeto e execução, gerando deficiência na interface de ligamento da placa com o substrato (Silva, 2014). Entretanto, conforme Bauer et al. (2015), a longo prazo, o descolamento surge devido fadiga causada pelas as variações dimensionais originadas pela radiação solar, presença de umidade ou movimentação estrutural do edifício.

As fissuras são geralmente as anomalias mais documentadas na literatura, pois estas permitem o ingresso de umidade e indicam uma descontinuidades no sistema. Na Figura 4 são mostrados os valores dos níveis de degradação referente à fissuração de acordo com a idade de cada revestimento. 

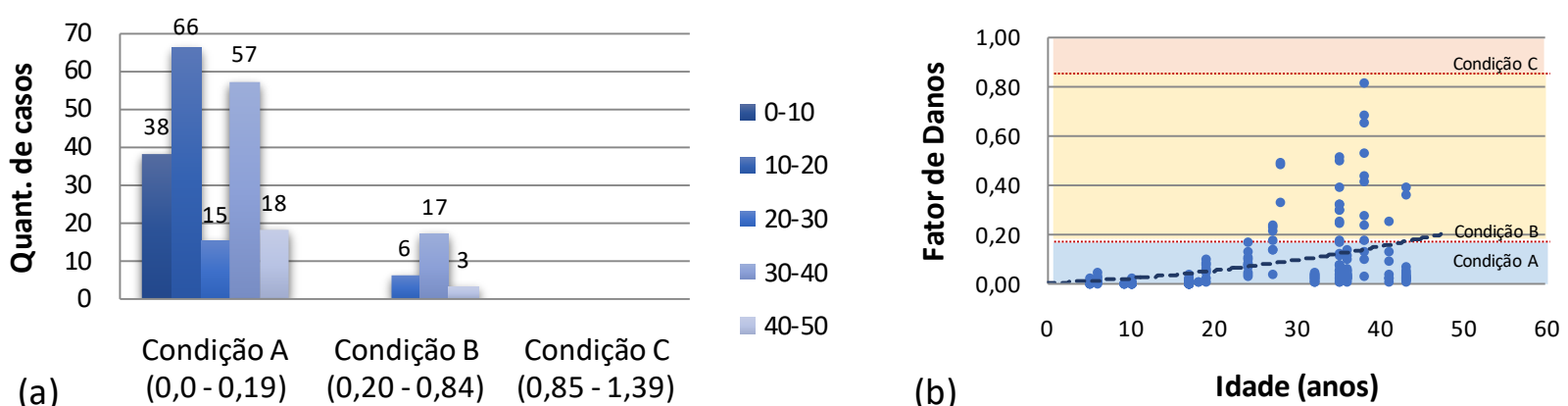

(b)

Idade (anos)

Figura 4 - Níveis de condição referente a fissuração: (a) Ocorrência de casos por faixa de idade e (b) Fator de Danos ao longo do tempo.

A condição A é a condição que apresenta maior quantidade de casos, o que indica que a maioria dos casos apresentam valores de FD de fissuração inferior a 0,19. Entretanto, contrário ao esperado, há uma quantidade elevada de casos nas idades entre 30 e 40 anos com FD de fissuração na condição A. Este fato pode ser justificado pelo fato da dificuldade de identificação de fissuras em sistemas de revestimento cerâmico. Muitas vezes as fissuras ocorrem internamente e só são percebidas quando esta facilita o surgimento de outra anomalias, tal como descolamento cerâmico. Diante disto, torna-se perceptivel a importancia da manutenção preventiva, em que são realizadas inspeções periódicas e que evitam a evolução da extensão e gravidade das anomalias.

A falhas no rejunte geralmente ocorrem por conta da incapacidade do rejunte em absorver deformações, resultando em aberturas que permitem o ingresso da umidade no sistema de revestimento e no não cumprimento das funções do sistema de revestimento das fachadas. Os valores dos níveis de degradação referentes à falha de rejunte são mostrados na Figura 5 de acordo com a idade de cada revestimento.
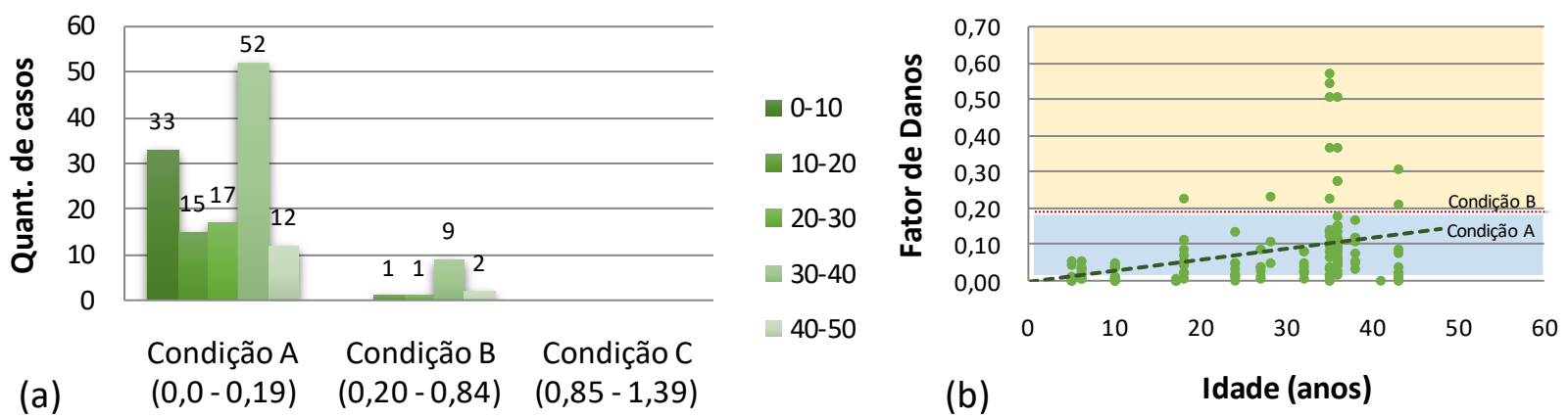

Figura 5 - Níveis de condição referente a falha de rejunte: (a) Ocorrência de casos por faixa de idade e (b) Fator de Danos ao longo do tempo.

Embora a quantidade de casos em que apresentam falha de rejunte seja inferior a quantidade de casos de descolamento cerâmico, o comportamento em relação às condições são semelhantes. A falha de rejunte é uma anomalia que afeta pouco a função estética da fachada, entretanto a presença desta prejudica a função de impermeabilidade e facilita o surgimento de outras anomalias, tal como descolamento cerâmico e a eflorescência. Segundo Souza (2019), na Condição A são sugeridas a realização de manutenção preventiva.

As eflorescências são manchas brancas formadas pela deposição de sais oriundos da alvenaria ou do próprio revestimento. A Figura 6 mostra os valores dos níveis de degradação referente a eflorescência de acordo com a idade de cada revestimento. 

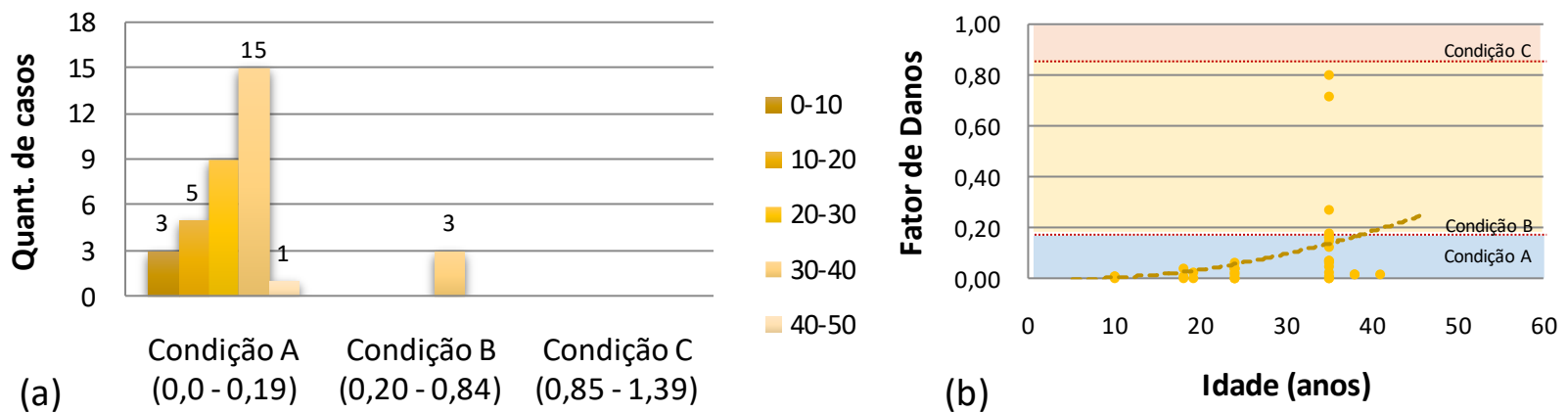

(b) Idade (anos)

Figura 6 - Níveis de condição referente a eflorescência: (a) Ocorrência de casos por faixa de idade e (b) Fator de Danos ao longo do tempo.

A eflorescência é uma das anomalias pouco notada em sistemas de revestimento cerâmico devido ao fato de esta ser uma considerada mancha e, esteticamente, incomodar pouco aos usuários. Entretanto, é importante destacar que dentre os diversos tipo de manchas que podem surgir em sistemas de revestimento de fachada, a eflorecência indica que há ou que houve a presença de água no sistema e, consequentemente, a função de impermeabilidade foi afetada.

O sistema de revestimento cerâmico tem sido amplamente utilizado devido às suas inúmeras vantagens, dentre elas destaca-se a resistência mecânica e a facilidade de manutenção (Souza, 2016). Entretanto é natural que com o passar do tempo qualquer sistema construtivo se degrade e apresente anomalias.

A norma brasileira de manutenção NBR 5674 (2012) indica períodos mínimos para tomada de ações de manutenção, seja esta preditiva e/ou inspeção de fachadas. O entendimento das anomalias e do comportamento destas ao longo do tempo permitem que sejam feitas recomendações de periodicidade de ações de manutenção. $O$ Quadro 2 apresenta as possiveis ações de manutenção e a periodicidade que estas devem ser feitas para as anomalias comuns em sistema de revestimento cerâmico de fachadas.

Quadro 2 - Periodicidade e possíveis ações de manutenção para cada anomalia

\begin{tabular}{|c|l|c|}
\hline Anomalia & \multicolumn{1}{|c|}{ Ação de manutenção } & Periodicidade \\
\hline $\begin{array}{c}\text { Descolamento } \\
\text { cerâmico }\end{array}$ & $\begin{array}{l}\text { Preventiva: Verificar a integridade da fachada e efetuar a } \\
\text { substituição de placas visualmente defeituosas. } \\
\text { Preditiva: Realizar inspeção para identificação de } \\
\text { cerâmicas com falha de aderência e fazer reconstituição. } \\
\text { Corretiva: Reconstituir as áreas em que apresentam } \\
\text { ausência e/ou empolamento de cerâmicas. }\end{array}$ & A cada seis meses \\
\hline Fissuração & $\begin{array}{l}\text { Preventiva: Verificar a envoltórias de aberturas e } \\
\text { substituição de placas com excesso de fissuras. } \\
\text { Preditiva: Realizar inspeção ao longo dos elementos } \\
\text { estruturais e efetuar a substituição de placas defeituosas. } \\
\text { Corretiva: Realizar inspeção completa da fachada e } \\
\text { efetuar substituição das placas defeituosas. }\end{array}$ & A cada três \\
\hline Falha de \\
Rejunte & $\begin{array}{l}\text { Preventiva: Verificar a integridade do rejunte e } \\
\text { reconstituir os que apresentarem defeitos. } \\
\text { Preditiva: Efetuar lavagem da fachada e reconstituir os } \\
\text { rejuntes que apresentam defeitos. } \\
\text { Corretiva: Reconstituição dos rejuntamentos e juntas de } \\
\text { movimentação. }\end{array}$ & A cada três anos \\
\hline Eflorescência & $\begin{array}{l}\text { Preventiva: Verificar a integridade dos peitoris. } \\
\text { Preditiva: Efetuar lavagem da fachada. } \\
\text { Corretiva:Verificar os locais de entrada de água e realizar } \\
\text { a impermeabilização. }\end{array}$ & Anual \\
\hline A cada dois anos \\
A cada três anos \\
\hline
\end{tabular}

As ações de manutenção sugeridas garantem o cumprimento da vida útil e podem até mesmo prolongar a vida útil so sistema de revestimento de fachada e, consequentemente, da edificação. Para que seja 
estendida a vida útil, é importante que a realização das ações de manutenção sejam feita por profissionais qualificados e seja realizado da forma correta. Sendo assim, não é recomendado o uso de máquina de alta pressão de água e produtos químicos que prejudiquem o sistema. Ainda que haja as ações de manutenção, é importante destacar que as ações dos usuários devem ser controladas, pois alterações na fachada podem facilitar o surgimento de anomalias. Cita-se como exemplo a perfuração do sistema para instalação de telas e ar condicionado.

\section{Conclusões}

O Método de Mensuração de Degradação, adotado nesta pesquisa, permitiu a obtenção de indicadores de degração e, consequentemente, diversas análises. A relação da extensão de degradação com o nível de condição foi analisado e permitiu realizar recomendações de periodicidade de ações de manutenção, considerando como os níveis de condição das anomalias podem afetar a funcionalidade e durabilidade do sistema.

Devido à analise do Fator de Danos percebeu-se que a grande parte dos casos encontradas nos primeiro anos das edificações em cada um dos tipos de anomalias são classificados como pertencente a condição $A$. Isso significa que são casos que não prejudicam a durabilidade do sistema. Os horizontes temporais de tomada de ações de manutenção anuais, bienais e a cada três anos que foram listadas podem facilmente garantir o cumprimento ou mesmo permitir o prolongamento da vida útil do sistema.

Entretando um resultado interessante que foi observado é que para o descolamento cerêmico tem-se mais casos acima da condição $A$ já nos primeiros anos da edificação. Uma vez que estes descolamentos são de grande probabilidade de gerar danos pessoais e materiais, foi recomendado que as primeiras ações de manutenção fossem tomadas mais cedo.

\section{Agradecimentos}

Os autores agradecem o apoio da Universidade de Brasília e do Instituto de Educação Superior de Brasília e ao grupo de pesquisa do programa de pós graduação em estruturas e construção civil da Universidade de Brasília, que investiga degradação em fachadas, pela a disponibilização das informações fornecidas.

\section{Referências Bibliográficas}

Associação Brasileira de Normas Técnicas (2013). NBR 15557-1: Desempenho de edificações habitacionais. Rio de Janeiro.

Associação Brasileira de Normas Técnicas (2012). NBR 5674: Manutenção de edificações - Procedimentos. Rio de Janeiro.

Bauer, E.; Castro, E. K.; Silva, M. N. B. (2015). Estimativa da degradação de fachadas com revestimento cerâmico: estudo de caso de edifícios de Brasília.Cerâmica, São Paulo , v. 61, n. 358, p. 151-159, jun. 2015. Disponível em: <https://doi.org/10.1590/0366-69132015613581786>. acesso em: 29 dez. 2020.

Duling, J.M.; Horak, E.; Cloete, C. (2008) Service life prediction beyond the "Factor Method". International Conference on Durability of Buildings Materials and Components - DBMC, Istambul, Turquia. pp. T42.

International Organization for Standardization (2017). ISO 15686-7: Buildings and constructed assets Service life planning - Part 7: Performance evaluation for feedback of service life data from practice. Geneva, Switzerland.

Piazzarollo, C.; Souza J. S.; Bauer, E. (2019). Aplicação do Método de mensuração de Degradação em revestimentos de Fachadas. 2o Workshop de Tecnologia de Tecnologia de Processos e Sistemas Construtivos. São Paulo, Brazil.

Pinheiro, P. I. S., Bauer, E. e Souza, J. S. (2017). Aplicação do Método de Mensuração da Degradação com a finalidade de quantificação da vida útil. Simpósio de argamassas e soluções térmicas - SBTA. p. 1-8. 
Silva, M. N. B.; Bauer, E.; Castro, E. K. (2014). Avaliação da degradação em sistemas de revestimento cerâmico de fachadas de brasília. In: Simpósio de Argamassa e soluções térmicas de revestimento, Anais..., p. 1-11(b).

Souza, J. S. (2016) Evolução da degradação de fachadas - efeito dos agentes de degradação e dos elementos constituintes. Dissertação de Mestrado. Universidade de Brasília, Brasília, Brasil. 114p.

Souza, J. S.; Silva, A.; De Brito, J.; Bauer, E. (2018) Analysis of the influencing factors of external wall ceramic claddings' service life using regression techniques. Engineering Failure Analysis. v.83, pp. 141-155.

Souza, J. S. (2019) Impacto dos fatores de degradação sobre a vida útil de fachadas de edifícios. Tese para obtenção de Grau de Doutor em Engenharia Civil. Universidade de Brasília, Brasília, Brasil. Disponível em: <https://repositorio.unb.br/bitstream/10482/38369/1/2019_J\%C3\%A9ssicaSiqueiradeSouza.pdf>. Acesso em: 22/12/2020. 